\title{
Sharpening and generalizations of Shafer-Fink and Wilker type inequalities: a new approach
}

Marija Rašajski, Tatjana Lutovac, Branko Malešević*

School of Electrical Engineering, University of Belgrade, Bulevar kralja Aleksandra 73, 11000 Belgrade, Serbia.

Communicated by S. H. Wu

\begin{abstract}
In this paper, we propose and prove some generalizations and sharpenings of certain inequalities of Wilker's and ShaferFink's type. Application of the Wu-Debnath theorem enabled us to prove some double sided inequalities.
\end{abstract}

Keywords: Sharpening, generalization, inequalities of Wilker's and Shafer-Fink's type.

2010 MSC: 33B10, 26D05.

(C)2018 All rights reserved.

\section{Introduction}

The main topic of this paper is the refinement and generalization of some inequalities of Wilker's and Shafer-Fink's type.

Wilker's inequality is an inequality of the following form

$$
\left(\frac{\sin x}{x}\right)^{2}+\frac{\tan x}{x}>2
$$

and holds for $x \in\left(0, \frac{\pi}{2}\right)$, [18].

That inequality had a great impact on numerous papers that address the theory of analytical inequalities [2].

Concerning Wilker's inequality, in this paper we propose and prove some extensions of Theorem 2.1 from [15], see also [14].

Shafer-Fink's inequality is the following double-sided inequality

$$
\frac{3 x}{2+\sqrt{1-x^{2}}}<\arcsin x<\frac{\pi x}{2+\sqrt{1-x^{2}}}
$$

and holds for $x \in(0,1)$, $[6,13,17]$.

\footnotetext{
*Corresponding author

Email addresses: marija.rasajski@etf.rs (Marija Rašajski), tatjana.lutovac@etf.rs (Tatjana Lutovac),

branko.malesevic@etf.rs (Branko Malešević)
}

doi: $10.22436 /$ jnsa.011.07.02

Received: 2018-03-30 Revised: 2018-04-15 Accepted: 2018-04-18 
The above-mentioned inequality also had a great impact on many papers in the theory of analytical inequalities [2]. It is important to say that inequalities of this type have applications in various fields of engineering $[1,5,16]$; see also $[4,10,12]$.

In this paper, concerning Shafer-Fink's inequality, we propose and prove some extensions of Theorems 1 and 2 from [11].

We now state the Wu-Debnath theorem (Theorem 2 in [19]), used in our proofs.

Theorem WD. Suppose that $\mathrm{f}(\mathrm{x})$ is a real function on $(\mathrm{a}, \mathrm{b})$, and that $\mathrm{n}$ is a positive integer such that

$$
f^{(k)}(a+), f^{(k)}(b-),(k \in\{0,1,2, \ldots, n\})
$$

exist.

(i) Supposing that $(-1)^{(n)} f^{(n)}(x)$ is increasing on $(a, b)$, then for all $x \in(a, b)$ the following inequality holds

$$
\begin{aligned}
\sum_{k=0}^{n-1} \frac{f^{(k)}(b-)}{k !}(x-b)^{k} & +\frac{1}{(a-b)^{n}}\left(f(a+)-\sum_{k=0}^{n-1} \frac{(a-b)^{k} f^{(k)}(b-)}{k !}\right)(x-b)^{n} \\
& <f(x)<\sum_{k=0}^{n} \frac{f^{(k)}(b-)}{k !}(x-b)^{k}
\end{aligned}
$$

Furthermore, if $(-1)^{n} f^{(n)}(x)$ is decreasing on $(a, b)$, then the reversed inequality of (1.1) holds.

(ii) Supposing that $\mathrm{f}^{(\mathrm{n})}(\mathrm{x})$ is increasing on $(\mathrm{a}, \mathrm{b})$, then for all $\mathrm{x} \in(\mathrm{a}, \mathrm{b})$ the following inequality also holds

$$
\begin{aligned}
\sum_{k=0}^{n-1} \frac{f^{(k)}(a+)}{k !}(x-a)^{k} & +\frac{1}{(b-a)^{n}}\left(f(b-)-\sum_{k=0}^{n-1} \frac{(b-a)^{k} f^{(k)}(a+)}{k !}\right)(x-a)^{n} \\
& >f(x)>\sum_{k=0}^{n} \frac{f^{(k)}(a+)}{k !}(x-a)^{k}
\end{aligned}
$$

Furthermore, if $\mathrm{f}^{(\mathrm{n})}(\mathrm{x})$ is decreasing on $(\mathrm{a}, \mathrm{b})$, then the reversed inequality of (1.2) holds.

Let us mention that an interesting application of Theorem WD to the inequalities that involve hyperbolic functions was considered in [8].

Here, we prove a statement that represents a natural extension of the above theorem.

Theorem 1.1. For the function $f:(a, b) \longrightarrow R$ let there exist the power series expansion

$$
f(x)=\sum_{k=0}^{\infty} c_{k}(x-a)^{k}
$$

for every $x \in(a, b)$, where $\left\{c_{k}\right\}_{k \in N_{0}}$ is the sequence of coefficients such that there is only a finite number of negative coefficients, and their indices are all in the set $\mathrm{J}=\left\{\mathrm{j}_{0}, \ldots, \mathrm{j}_{\ell}\right\}$. Then, for the function

$$
F(x)=f(x)-\sum_{i=0}^{\ell} c_{j_{i}}(x-a)^{j_{i}}=\sum_{k \in N_{0} \backslash J} c_{k}(x-a)^{k},
$$

and the sequence $\left\{\mathrm{C}_{\mathrm{k}}\right\}_{\mathrm{k} \in \mathrm{N}_{0}}$ of the non-negative coefficients defined by

$$
C_{k}=\left\{\begin{array}{cl}
c_{k}: & c_{k}>0 \\
0 & : c_{k} \leqslant 0
\end{array}\right.
$$

holds that

$$
F(x)=\sum_{k=0}^{\infty} C_{k}(x-a)^{k}
$$


for every $x \in(a, b)$.

It is also $\mathrm{F}^{(\mathrm{k})}(\mathrm{a}+)=\mathrm{k} ! \mathrm{C}_{\mathrm{k}}(\mathrm{k} \in\{0,1,2, \ldots, \mathrm{n}\})$ and the following inequalities hold

$$
\begin{aligned}
& \sum_{k=0}^{n-1} C_{k}(x-a)^{k}+ \frac{1}{(b-a)^{n}}\left(F(b-)-\sum_{k=0}^{n-1}(b-a)^{k} C_{k}\right)(x-a)^{n} \\
&>F(x)>\sum_{k=0}^{n} C_{k}(x-a)^{k}
\end{aligned}
$$

i.e.,

$$
\begin{gathered}
\sum_{k=0}^{n-1} c_{k}(x-a)^{k}+\sum_{i=0}^{\ell} c_{j_{i}}(x-a)^{j_{i}}+\frac{(x-a)^{n}}{(b-a)^{n}}\left(f(b-)-\sum_{k=0}^{n-1} c_{k}(b-a)^{k}-\sum_{i=0}^{\ell} c_{j_{i}}(b-a)^{j_{i}}\right) \\
>f(x)>\sum_{k=0}^{n} c_{k}(x-a)^{k}+\sum_{i=0}^{\ell} c_{j_{i}}(x-a)^{j_{i}}
\end{gathered}
$$

for every $x \in(a, b)$

Proof. This is a direct consequence of the previous theorem. The fact that all coefficients are positive implies that all derivatives are positive, and, consequently, corresponding functions are increasing.

Corollary 1.2. Let there hold the conditions from the previous theorem. If

$$
\mathrm{n}>\max \left\{\mathbf{j}_{0}, \ldots, \mathbf{j}_{\ell}\right\},
$$

then the following holds

$$
\sum_{k=0}^{n-1} c_{k}(x-a)^{k}+\frac{1}{(b-a)^{n}}\left(f(b-)-\sum_{k=0}^{n-1} c_{k}(b-a)^{k}\right)(x-a)^{n}>f(x)>\sum_{k=0}^{n} c_{k}(x-a)^{k}
$$

for every $x \in(a, b)$.

\section{Main results}

\subsection{Wilker's type inequalities}

The following statement was proved in [15]:

Theorem 2.1 ([15, Theorem 2.1$])$. For every $x \in\left(0, \frac{\pi}{2}\right)$ the following inequalities are true

$$
2+\left(\frac{8}{45}-a(x)\right) x^{3} \tan x<\left(\frac{\sin x}{x}\right)^{2}+\frac{\tan x}{x}<2+\left(\frac{8}{45}-b_{1}(x)\right) x^{3} \tan x,
$$

where $\mathrm{a}(\mathrm{x})=\frac{8}{945} \mathrm{x}^{2}, \mathrm{~b}_{1}(\mathrm{x})=\frac{8}{945} \mathrm{x}^{2}-\frac{\mathrm{a}}{14175} \mathrm{x}^{4}$ and $\mathrm{a}=\frac{480 \pi^{6}-40320 \pi^{4}+3628800}{\pi^{8}}=17.15041 \ldots$

Above theorem is also an extension of Theorem 1 from [14].

Let us notice that the inequality (2.1) could be stated as an equivalent inequality of the following form

$$
\frac{8}{45} x^{3}-\frac{8}{945} x^{5}<\frac{1}{x}+\frac{\sin 2 x}{2 x^{2}}-2 \cot x<\frac{8}{45} x^{3}-\frac{8}{945} x^{5}+\frac{a}{14175} x^{7}
$$

for $x \in\left(0, \frac{\pi}{2}\right)$.

In this paper, we sharpen the previous double-sided inequality using Theorem WD. 
Theorem 2.2. For the function

$$
f(x)=\frac{1}{x}+\frac{\sin 2 x}{2 x^{2}}-2 \cot x-\frac{8 x^{3}}{45}+\frac{8 x^{5}}{945}
$$

where $x \in\left(0, \frac{\pi}{2}\right)$, the following sequence of inequalities holds

$$
\sum_{k=0}^{m} c_{k} x^{2 k+1}<f(x)<\sum_{k=0}^{m-1} c_{k} x^{2 k+1}+\left(f\left(\frac{\pi}{2}\right)-\sum_{k=0}^{m-1} c_{k}\left(\frac{\pi}{2}\right)^{2 k+1}\right)\left(\frac{2 x}{\pi}\right)^{2 m+1}
$$

for $x \in\left(0, \frac{\pi}{2}\right)$ and $\mathrm{m} \in \mathrm{N}$ and $\mathrm{c}_{0}=\mathrm{c}_{1}=\mathrm{c}_{2}=0$ and for $\mathrm{k} \geqslant 3$

$$
c_{k}=\frac{2^{2 k+2}\left((4 k+6)\left|\mathbf{B}_{2 k+2}\right|+(-1)^{k+1}\right)}{(2 k+3) !}
$$

where $\mathbf{B}_{\boldsymbol{i}}$ are Bernoulli's numbers.

Proof. First, let us recall some well-known series expansions

$$
\sin 2 x=\sum_{k=1}^{\infty} \frac{(-1)^{k} 2^{2 k-1}}{(2 k-1) !} x^{2 k-1} \quad(x \in R)
$$

and

$$
\cot x=\frac{1}{x} \sum_{k=0}^{\infty} \frac{\left|\mathbf{B}_{2 k}\right| 2^{2 k}}{(2 k) !} x^{2 k} \quad(x \in(-\pi, 0) \cup(0, \pi)) .
$$

If we define $f(0)=0$, then we have Taylor's expansion of the function $f(x)$ for $x=0$

$$
f(x)=\sum_{k=0}^{\infty} c_{k} x^{2 k+1}
$$

where $c_{0}=c_{1}=c_{2}=0$ and for $\mathrm{k} \geqslant 3$

$$
c_{k}=\frac{2^{2 k+2}\left((4 k+6)\left|\mathbf{B}_{2 k+2}\right|+(-1)^{k+1}\right)}{(2 k+3) !} .
$$

The obtained Taylor's expansion of the function $f(x)$ converges for $x \in\left(0, \frac{\pi}{2}\right)$.

Based on (2.3), it is evident that for the sequence

$$
c_{3}=\frac{16}{14175}, c_{4}=\frac{8}{467775}, c_{5}=\frac{3184}{638512875}, \ldots
$$

holds that $c_{k}>0$ for $k \geqslant 3$. Then, the function

$$
f(x)=\frac{16 x^{7}}{14175}+\frac{8 x^{9}}{467775}+\frac{3184 x^{11}}{638512875}+\frac{272 x^{13}}{638512875}+\frac{7264 x^{15}}{162820783125}+\ldots,
$$

for $x \in\left(0, \frac{\pi}{2}\right)$, satisfies the condition:

$$
f^{(n)}(x)>0
$$

for $x \in\left(0, \frac{\pi}{2}\right)$ and $n \in N$. Then, for $n \in N$, the functions $f^{(n)}(x)$ are all increasing for $x \in\left(0, \frac{\pi}{2}\right)$, and there exist values $f\left(\frac{\pi}{2}-\right), f^{(k)}(0+)$ for every $k \in\{0,1, \ldots, n\}$.

The right-hand side of the inequality (2.2) is obtained using Theorem WD. 
Example 2.3. Now, let us show several examples of approximations of the function $f(x)$ obtained for $\mathrm{m}=3,4,5,6$ and $x \in\left(0, \frac{\pi}{2}\right)$ :

- For $m=3$ we get the double-sided inequality that was proved in [15]:

$$
\frac{16}{14175} x^{7}<f(x)<\left(\frac{2}{\pi}\right)^{7}\left(\frac{2}{\pi}-\frac{\pi^{3}}{45}+\frac{\pi^{5}}{3780}\right) x^{7}
$$

and, in this way, a new proof of the results from the paper [15] was obtained.

For $m>3$ the results that follow are higher accuracies.

- For $\mathrm{m}=4$ we have :

$$
\frac{16 x^{7}}{14175}+\frac{8 x^{9}}{467775}<f(x)<\frac{16 x^{7}}{14175}+\left(\frac{2}{\pi}\right)^{9}\left(\frac{2}{\pi}-\frac{\pi^{3}}{45}+\frac{\pi^{5}}{3780}-\frac{\pi^{7}}{113400}\right) x^{9} .
$$

- For $m=5$ we have:

$$
\begin{aligned}
\frac{16 x^{7}}{14175}+\frac{8 x^{9}}{467775}+\frac{3184 x^{11}}{638512875}<f(x)< & \frac{16 x^{7}}{14175}+\frac{8 x^{9}}{467775} \\
& +\left(\frac{2}{\pi}\right)^{11}\left(\frac{2}{\pi}-\frac{\pi^{3}}{45}+\frac{\pi^{5}}{3780}-\frac{\pi^{7}}{113400}-\frac{\pi^{9}}{29937600}\right) x^{11}
\end{aligned}
$$

- For $\mathrm{m}=6$ we have:

$$
\begin{aligned}
& \frac{16 x^{7}}{14175}+\frac{8 x^{9}}{467775}+\frac{3184 x^{11}}{638512875}+\frac{272 x^{13}}{638512875}<f(x)< \\
& <\frac{16 x^{7}}{14175}+\frac{8 x^{9}}{467775}+\frac{3184 x^{11}}{638512875}+\left(\frac{2}{\pi}\right)^{11}\left(\frac{2}{\pi}-\frac{\pi^{3}}{45}+\frac{\pi^{5}}{3780}-\frac{\pi^{7}}{113400}-\frac{\pi^{9}}{29937600}-\frac{199 \pi^{11}}{81729648000}\right) x^{13} .
\end{aligned}
$$

Remark 2.4. Let us note that Theorem WD enables us to estimate the error of approximation. The difference between the right-hand side and the left-hand side of the double-sided inequality in the previous theorem can be represented by the following function

$$
R_{m}(x)=\left(f\left(\frac{\pi}{2}\right)-\sum_{k=3}^{m} \frac{2^{2 k+2}\left((4 k+6)\left|B_{2 k+2}\right|+(-1)^{k+1}\right)}{(2 k+3) !}\left(\frac{\pi}{2}\right)^{2 k+1}\right)\left(\frac{2 x}{\pi}\right)^{2 m+1}
$$

for $x \in\left(0, \frac{\pi}{2}\right)$.

The maximum values of the above-mentioned difference in the interval $\left(0, \frac{\pi}{2}\right)$, for $m=3,4,5,6$, are shown in the table below

\begin{tabular}{|c|c|c|c|c|}
\hline$m$ & 3 & 4 & 5 & 6 \\
\hline$R_{m}(x)$ & 0.00191501 & 0.000919303 & 0.000202959 & 0.0000519655 \\
\hline
\end{tabular}

\subsection{Shafer-Fink's type inequalities}

Let us start from the following assertions proved by Bercu in [3].

Statement 1 ([3, Theorem 1$])$. For every real number $0 \leqslant x \leqslant 1$, the following two-sided inequality holds

$$
\frac{x^{5}}{180}+\frac{x^{7}}{189} \leqslant \arcsin x-\frac{3 x}{2+\sqrt{1-x^{2}}} \leqslant \frac{\pi-3}{2} .
$$


Statement 2 ([3, Theorem 3]). For every $x \in[0,1]$ on the left-hand side and every $x \in[0,0.871433]$ on the right-hand side, the following inequalities hold true

$$
\left(1-\frac{\pi}{3}\right) x+\left(\frac{1}{6}-\frac{\pi}{18}\right) x^{3} \leqslant \arcsin x-\frac{\pi x}{2+\sqrt{1-x^{2}}} \leqslant\left(1-\frac{\pi}{3}\right) x .
$$

Statement 3 ([3, Theorem 2]). For every $0 \leqslant x \leqslant 1$, one has

$$
\arcsin x-\frac{3 x}{2+\sqrt{1-x^{2}}} \geqslant \frac{a(x)}{2+\sqrt{1-x^{2}}},
$$

where $\mathrm{a}(\mathrm{x})=(1 / 60) \mathrm{x}^{5}+(11 / 840) \mathrm{x}^{7}$.

In [11], the authors proved the following theorem.

Statement 4 ([11, Theorem 1]). For $x \in[0,1], n \in N$ and $k=3 \vee k=\pi$ the following inequality holds

$$
\sum_{m=0}^{n} D_{k}(m) x^{2 m+1} \leqslant \arcsin x-\frac{k x}{2+\sqrt{1-x^{2}}}
$$

where

$$
D_{k}(m)=\frac{(2 m) !}{(m !)^{2}(2 m+1) 2^{2 m}}-\left(\frac{(-1)^{m} k}{3^{m+1}}+\sum_{i=0}^{m-1} \frac{k(-1)^{m-1-i}(2 i) !}{3^{m-i} i !(i+1) ! 2^{2 i+1}}\right)>0
$$

for $m \in N_{0}, m \geqslant 2\left(D_{k}(0)=D_{k}(1)=0\right)$.

Remark 2.5. For $n=3, k=3$ and $n=1, k=\pi$ we get the left-hand sides of the inequalities stated in Theorems 1 and 3 from [3] by G. Bercu.

Now, let us consider the functions

$$
f_{k}(x)=\arcsin x-\frac{k x}{2+\sqrt{1-x^{2}}}
$$

for $x \in[0,1]$, and $k=3 \vee k=\pi$. Then, using Theorem $W D$, we get:

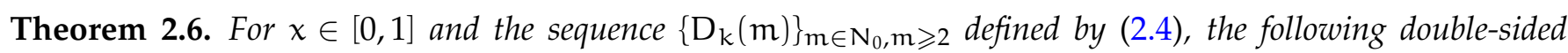
inequalities hold true

$$
\sum_{m=0}^{n} D_{k}(m) x^{2 m+1} \leqslant f_{k}(x) \leqslant \sum_{m=0}^{n-1} D_{k}(m) x^{2 m+1}+\left(f_{k}(1)-\sum_{m=0}^{n-1} D_{k}(m)\right) x^{2 n+1} .
$$

Example 2.7. We show a few examples of approximations of the function $f_{k}(x)$ for $k=3, n=3,4,5,6$ and $x \in[0,1]:$

- For $n=3$ we have:

$$
\frac{x^{5}}{180}+\frac{x^{7}}{189} \leqslant f_{3}(x) \leqslant \frac{x^{5}}{180}+\left(-\frac{271}{180}+\frac{\pi}{2}\right) x^{7}
$$

- For $\mathrm{n}=4$ we have:

$$
\frac{x^{5}}{180}+\frac{x^{7}}{189}+\frac{23 x^{9}}{5184} \leqslant f_{3}(x) \leqslant \frac{x^{5}}{180}+\frac{x^{7}}{189}+\left(-\frac{5711}{3780}+\frac{\pi}{2}\right) x^{9} .
$$

- For $n=5$ we have:

$$
\frac{x^{5}}{180}+\frac{x^{7}}{189}+\frac{23 x^{9}}{5184}+\frac{629 x^{11}}{171072} \leqslant f_{3}(x) \leqslant \frac{x^{5}}{180}+\frac{x^{7}}{189}+\frac{23 x^{9}}{5184}+\left(-\frac{274933}{181440}+\frac{\pi}{2}\right) x^{11} .
$$


- For $n=6$ we have:

$$
\frac{x^{5}}{180}+\frac{x^{7}}{189}+\frac{23 x^{9}}{5184}+\frac{629 x^{11}}{171072}+\frac{14929 x^{13}}{4852224} \leqslant f_{3}(x) \leqslant \frac{x^{5}}{180}+\frac{x^{7}}{189}+\frac{23 x^{9}}{5184}+\frac{629 x^{11}}{171072}+\left(-\frac{2273701}{1496880}+\frac{\pi}{2}\right) x^{13} .
$$

Example 2.8. Now, let us present several examples of approximations of the function $f_{k}(x)$ for $k=\pi$, $\mathrm{n}=3,4,5,6$ and $x \in[0,1]$ :

- For $\mathrm{n}=3$ we have:

$$
\begin{gathered}
\left(1-\frac{\pi}{3}\right) x+\left(\frac{1}{6}-\frac{\pi}{18}\right) x^{3}+\left(\frac{3}{40}-\frac{5 \pi}{216}\right) x^{5}+\left(\frac{5}{112}-\frac{17 \pi}{1296}\right) x^{7} \leqslant f_{\pi}(x) \leqslant \\
\leqslant\left(1-\frac{\pi}{3}\right) x+\left(\frac{1}{6}-\frac{\pi}{18}\right) x^{3}+\left(\frac{3}{40}-\frac{5 \pi}{216}\right) x^{5}+\left(\frac{149}{120}+\frac{89 \pi}{216}\right) \chi^{7} .
\end{gathered}
$$

- For $n=4$ we have:

$$
\begin{aligned}
& \left(1-\frac{\pi}{3}\right) x+\left(\frac{1}{6}-\frac{\pi}{18}\right) \chi^{3}+\left(\frac{3}{40}-\frac{5 \pi}{216}\right) x^{5}+\left(\frac{5}{112}-\frac{17 \pi}{1296}\right) x^{7}+\left(\frac{35}{1152}-\frac{269 \pi}{31104}\right) x^{9} \leqslant f_{\pi}(x) \leqslant \\
& \leqslant\left(1-\frac{\pi}{3}\right) x+\left(\frac{1}{6}-\frac{\pi}{18}\right) x^{3}+\left(\frac{3}{40}-\frac{5 \pi}{216}\right) x^{5}+\left(\frac{5}{112}-\frac{17 \pi}{1296}\right) x^{7}+\left(-\frac{2161}{1680}+\frac{551 \pi}{1296}\right) x^{9} .
\end{aligned}
$$

- For $n=5$ we have :

$$
\begin{gathered}
\left(1-\frac{\pi}{3}\right) x+\left(\frac{1}{6}-\frac{\pi}{18}\right) x^{3}+\left(\frac{3}{40}-\frac{5 \pi}{216}\right) x^{5}+\left(\frac{5}{112}-\frac{17 \pi}{1296}\right) x^{7}+\left(\frac{35}{1152}-\frac{269 \pi}{31104}\right) x^{9}+\left(\frac{63}{2816}-\frac{1163 \pi}{186624}\right) x^{11} \leqslant f_{\pi}(x) \leqslant \\
\leqslant\left(1-\frac{\pi}{3}\right) x+\left(\frac{1}{6}-\frac{\pi}{18}\right) x^{3}+\left(\frac{3}{40}-\frac{5 \pi}{216}\right) x^{5}+\left(\frac{5}{112}-\frac{17 \pi}{1296}\right) x^{7}+\left(\frac{35}{1152}-\frac{269 \pi}{31104}\right) x^{9}+\left(-\frac{53089}{40320}+\frac{13493 \pi}{31104}\right) \chi^{11} .
\end{gathered}
$$

- For $n=6$ we have:

$$
\begin{gathered}
\left(1-\frac{\pi}{3}\right) x+\left(\frac{1}{6}-\frac{\pi}{18}\right) x^{3}+\left(\frac{3}{40}-\frac{5 \pi}{216}\right) x^{5}+\left(\frac{5}{112}-\frac{17 \pi}{1296}\right) x^{7}+\left(\frac{35}{1152}-\frac{269 \pi}{31104}\right) x^{9}+\left(\frac{63}{2816}-\frac{1163 \pi}{186624}\right) x^{11}+\left(\frac{231}{13312}-\frac{10657 \pi}{2239488}\right) x^{13} \leqslant \\
\leqslant f_{\pi}(x) \leqslant \\
\leqslant\left(1-\frac{\pi}{3}\right) x+\left(\frac{1}{6}-\frac{\pi}{18}\right) x^{3}+\left(\frac{3}{40}-\frac{5 \pi}{216}\right) x^{5}+\left(\frac{5}{112}-\frac{17 \pi}{1296}\right) x^{7}+\left(\frac{35}{1152}-\frac{269 \pi}{31104}\right) x^{9}+\left(\frac{63}{2816}-\frac{1163 \pi}{186624}\right) x^{11}+\left(-\frac{1187803}{887040}+\frac{82121 \pi}{186624}\right) x^{13} .
\end{gathered}
$$

In [11] the authors proved the following assertion.

Statement 5 ([11, Theorem 2]). If $n \in N$ and $n \geqslant 2$, then

$$
\arcsin x-\frac{3 x}{2+\sqrt{1-x^{2}}} \geqslant \frac{\sum_{m=2}^{n} E(m) x^{2 m+1}}{2+\sqrt{1-x^{2}}}
$$

for every $x \in[0,1]$, where

$$
E(m)=\frac{m(2 m-1) !}{(2 m+1) 2^{2 m-2} m !^{2}}-\frac{2 m 2^{2 m-2}(m-1) !^{2}}{(2 m+1) !}>0
$$

for $m \in N, m \geqslant 2(E(1)=0)$.

Remark 2.9. For $n=3$ we get the left-hand sides of the inequality stated in Theorem 2 from [3] by G. Bercu.

Using Theorem WD we prove the following theorem. 


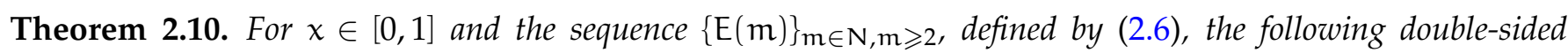
inequalities hold true:

$$
\frac{\sum_{m=2}^{n} E(m) x^{2 m+1}}{2+\sqrt{1-x^{2}}} \leqslant \arcsin x-\frac{3 x}{2+\sqrt{1-x^{2}}} \leqslant \frac{\sum_{m=2}^{n-1} E(m) x^{2 m+1}+\left(\pi-\sum_{m=0}^{n-1} E(m)\right) x^{2 n+1}}{2+\sqrt{1-x^{2}}} .
$$

Example 2.11. Following are several examples of approximations of the function

$$
\arcsin x-\frac{3 x}{2+\sqrt{1-x^{2}}}
$$

for $n=3,4,5,6$ and $x \in[0,1]$ :

- For $\mathrm{n}=3$ we have:

$$
\frac{\frac{1}{60} x^{5}+\frac{11}{840} x^{7}}{2+\sqrt{1-x^{2}}} \leqslant \arcsin x-\frac{3 x}{2+\sqrt{1-x^{2}}} \leqslant \frac{\frac{1}{60} x^{5}+\left(\pi-\frac{181}{60}\right) x^{7}}{2+\sqrt{1-x^{2}}}
$$

- For $\mathrm{n}=4$ we have:

$$
\frac{\frac{1}{60} x^{5}+\frac{11}{840} x^{7}+\frac{67}{6720} x^{9}}{2+\sqrt{1-x^{2}}} \leqslant \arcsin x-\frac{3 x}{2+\sqrt{1-x^{2}}} \leqslant \frac{\frac{1}{60} x^{5}+\frac{11}{840} x^{7}+\left(\pi-\frac{509}{168}\right) x^{9}}{2+\sqrt{1-x^{2}}} .
$$

- For $\mathrm{n}=5$ we have:

$$
\frac{\frac{1}{60} x^{5}+\frac{11}{840} x^{7}+\frac{67}{6720} x^{9}+\frac{3461}{443520} x^{11}}{2+\sqrt{1-x^{2}}} \leqslant \arcsin x-\frac{3 x}{2+\sqrt{1-x^{2}}} \leqslant \frac{\frac{1}{60} x^{5}+\frac{11}{840} x^{7}+\frac{67}{6720} x^{9}+\left(\pi-\frac{6809}{2240}\right) x^{11}}{2+\sqrt{1-x^{2}}} .
$$

- For $n=6$ we have:

$$
\begin{gathered}
\frac{\frac{1}{60} x^{5}+\frac{11}{840} x^{7}+\frac{67}{6720} x^{9}+\frac{3461}{443520} x^{11}+\frac{29011}{4612608} x^{13}}{2+\sqrt{1-x^{2}}} \leqslant \arcsin x-\frac{3 x}{2+\sqrt{1-x^{2}}} \leqslant \\
\leqslant \frac{\frac{1}{60} x^{5}+\frac{11}{840} x^{7}+\frac{67}{6720} x^{9}+\frac{3461}{443520} x^{11}+\left(\pi-\frac{1351643}{443520}\right) x^{13}}{2+\sqrt{1-x^{2}}} .
\end{gathered}
$$

\section{Conclusion}

In this paper, we proposed and proved new inequalities which represent refinements and generalizations of the inequalities stated in [11] related to Shafer-Fink's inequality for the inverse sine function, as well as the inequalities stated in [15] related to Wilker's inequality. Finally, let us note that proofs of inequalities (2.2) for any fixed $m \in N$, and inequalities (2.5) and (2.7) for any fixed $n \in N$, can be obtained by methods and algorithms developed in [9] and [7].

\section{Acknowledgment}

Research of the first and second and third author was supported in part by the Serbian Ministry of Education, Science and Technological Development, under Projects ON 174033, TR 32023 and ON 174032 \& III 4400, respectively. 


\section{References}

[1] G. Alirezaei, R. Mathar, Scrutinizing the average error probability for nakagami fading channels, in The IEEE International Symposium on Information Theory (ISIT'14), Honolulu, Hawai, USA, (2014), 2884-2888. 1

[2] D. G. Anderson, M. Vuorinen, X. Zhang, Topics in Special Functions III, Analytic number theory, approximation theory and special functions, 297-345, Springer, New York, (2014). 1

[3] G. Bercu, Sharp Refinements for the Inverse Sine Function Related to Shafer-Fink's Inequality, Math. Probl. Eng., 2017 (2017), 5 pages. 2.2, 1, 2, 3, 2.5, 2.9

[4] M. J. Cloud, B. C. Drachman, L. P. Lebedev, Inequalities With Applications to Engineering, Springer, London, (2014). 1

[5] G. T. F. De Abreu, Jensen-Cotes upper and lower bounds on the Gaussian Q-function and related functions, IEEE Trans. Commun., 57 (2009), 3328-3338. 1

[6] A. M. Fink, Two inequalities, Univ. Beograd Publ. Elektroteh. Fak. Ser. Mat., 6 (1995), 49-50. 1

[7] T. Lutovac, B. Malešević, C. Mortici, The natural algorithmic approach of mixed trigonometric-polynomial problems, J. Inequal. Appl., 2017 (2017), 16 pages. 3

[8] M. Makragić, A method for proving some inequalities on mixed hyperbolic-trigonometric polynomial functions, J. Math. Inequal., 11 (2017), 817-829. 1

[9] B. Malešević, M. Makragić, A Method for Proving Some Inequalities on Mixed Trigonometric Polynomial Functions, J. Math. Inequal., 10 (2016), 849-876. 3

[10] B. Malešević, T. Lutovac, M. Rašajski, C. Mortici, Extensions of the natural approach to refinements and generalizations of some trigonometric inequalities, Adv. Difference Equ., 2018 (2018), 15 pages. 1

[11] B. Malešević, M. Rašajski, T. Lutovac, Refinements and generalizations of some inequalities of Shafer-Fink's type for the inverse sine function, J. Inequal. Appl., 2017 (2017), 9 pages. 1, 2.2, 4, 2.2, 5, 3

[12] B. Malešević, M. Rašajski, T. Lutovac, Refined estimates and generalizations of inequalities related to the arctangent function and Shafer's inequality, arXiv, 2017 (2017), 15 pages. 1

[13] D. S. Mitrinović, Analytic Inequalities, Springer-Verlag, New York-Berlin, (1970). 1

[14] C. Mortici, A subtly analysis of Wilker inequation, Appl. Math. Comput., 231 (2014), 516-520. 1, 2.1

[15] M. Nenezić, B. Malešević, C. Mortici, New approximations of some expressions involving trigonometric functions, Appl. Math. Comput., 283 (2016), 299-315. 1, 2.1, 2.1, 2.3, 3

[16] G. Rahmatollahi, G. T. F. De Abreu, Closed-Form Hop-Count Distributions in Random Networks with Arbitrary Routing, IEEE Trans. Commun., 60 (2012), 429-444. 1

[17] R. E. Shafer, Elementary Problems 1867, Amer. Math. Monthly, 73 (1966), Page 309. 1

[18] J. B. Wilker, Elementary Problems 3306, Amer. Math. Monthly 96 (1989), Page 55. 1

[19] S. Wu, L. Debnath, A generalization of L'Hospital-type rules for monotonicity and its application, Appl. Math. Lett., 22 (2009), 284-290. 1 